\title{
ROMANIAN
}

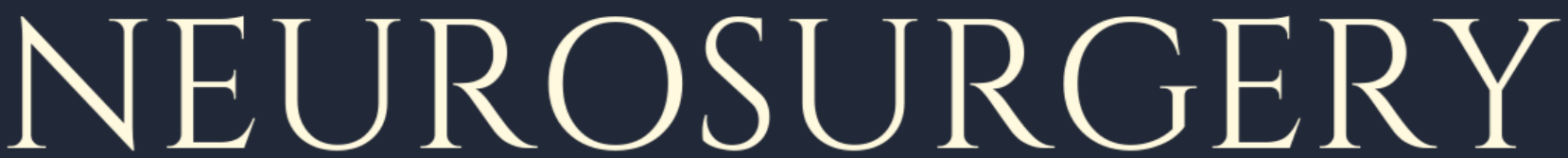

\author{
Vol. XXXIV | No. $1 \quad$ March 2020
}

\section{Management of facial paralysis following treatment of neurosurgical tumours}

\author{
Costan V. V., \\ Ciofu M. L., \\ Sava F., \\ Boisteanu O., \\ Dabija M.
}




\section{Management of facial paralysis following treatment of neurosurgical tumours}

\author{
Costan V.V. ${ }^{1}$, Ciofu M.L. ${ }^{1}$, Sava F. ${ }^{1}$, Boisteanu O. ${ }^{2}$, Dabija M. ${ }^{3}$ \\ 1 Faculty of Dental Medicine, Department of Surgery, Oral and \\ Maxillofacial Surgery, "Grigore T. Popa" University of Medicine and \\ Pharmacy, Iasi, ROMANIA \\ 2 Faculty of Dental Medicine, Department of Surgery, \\ Anaesthesiology, "Grigore T. Popa" University of Medicine and \\ Pharmacy, Iasi, ROMANIA \\ 3 Faculty of Medicine, Department of Surgery II, Neurosurgery, \\ "Grigore T. Popa" University of Medicine and Pharmacy, Iasi, RomanIA
}

\section{ABSTRACT}

The purpose of this study is to present our experience on improving the quality of life of patients with facial paralysis due to an operated intracranial tumour, by performing minimally invasive static reanimation procedures. We reviewed the clinical information pertaining to neurosurgical patients with facial paralysis that underwent static reanimation. The study included 11 patients with complete facial nerve paralysis of all nerve branches, that reported different primary complaints upon presentation. The performed procedures consisted of gold plate insertion into the superior eyelid, inferior eyelid ectropion correction or suture suspension. The functional results were favourable in all cases and the resulting appearance was acceptable. The choice of the different techniques used is discussed. Good outcomes are possible using static reanimation with an adequate adaptation of the techniques to the main patient complaint.

\section{INTRODUCTION}

Definitive facial nerve injury during neurosurgical procedures for benign or malignant tumors of the posterior and middle cranial fossae, cerebellopontine angle and lateral skull base, is often unavoidable, either due to existing preoperative tumor invasion or compression, or due to the proximity of the nerve, since the most important desiderate is avoiding tumor recurrence $(1,2)$.

Sequelae associated with facial nerve paralysis lead to important functional impairment as well as aesthetic changes comprising of a ptotic appearance of the involved hemiface with consequences regarding the emotional wellbeing of the patient and social integration. These factors cause a significant decline in the life quality of the patient $(3,4)$. Static reanimation procedures aim to restore facial symmetry, improve deglutition, mastication and phonation disturbances, while also decreasing complications that endanger vision $(5,6)$.

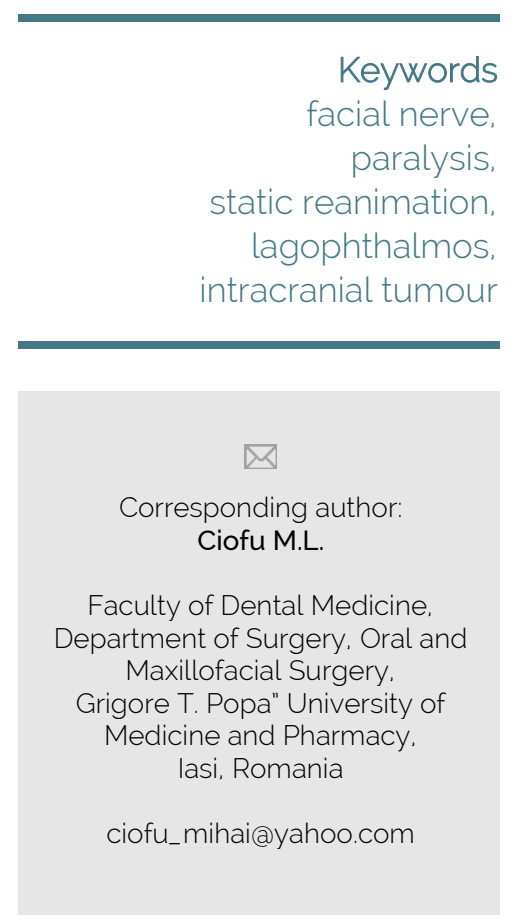

Copyright and usage. This is an Open Access article distributed under the terms of the Creative Commons Attribution Non-Commercial No Derivatives License (https://creativecommons org/licenses/by-nc-nd/4.0/) which permits noncommercial re-use, distribution, and reproduction in any medium, provided the original work is unaltered and is properly cited.

The written permission of the Romanian Society of Neurosurgery must be obtained for commercial re-use or in order to create a derivative work.

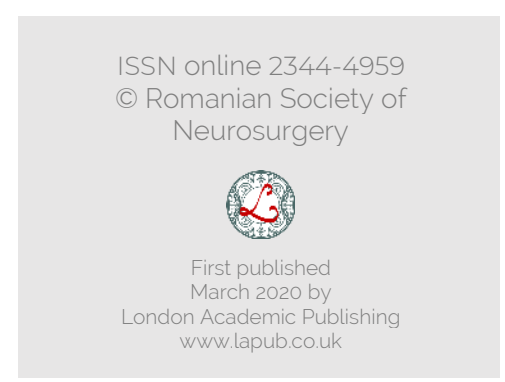


The particularity of patients with operated tumors involving the neurosurgical territory is that the nerve is injured along its intracranial route, making functional rehabilitation by nerve graft interposition practically impossible (1). Additionally, using the contralateral facial nerve branches carries the risk for bilateral injury. Therefore, the minimally invasive static reanimation procedures are highly indicated and can provide an increase in the quality of life of those patients without the need to perform major surgery.

In this manuscript we aim to present the benefits of different static facial reanimation procedures that we used in patients operated for neurosurgical tumors, in relation to the main complaints of the patients.

\section{MATERIAL AND METHODS}

We reviewed the medical charts of patients that presented for facial nerve paralysis following surgery for the removal of tumors in the neurosurgical territory. Only patients that underwent static reanimation procedures were included in the study. The minimum follow-up period was 6 months. The general information, the main complaints of the patients, the time passed from the onset of facial paralysis, the type of static reanimation procedure and the postoperative outcomes were all documented.

\section{RESULTS}

In total, 11 patients were included in the study, consisting of 6 men and 5 women, aged 46 to 72 years old. All patients had complete nerve paralysis of all facial nerve branches, showing all known signs of nerve paralysis in different degrees, including facial asymmetry, lagophthalmos, lower eyelid ectropion, brow ptosis, ptosis of the oral commissure with sialorrhea, deglutition, mastication and phonation disturbances. Still, the most bothersome complaint upon presentation was lagophthalmos in 6 patients, sialorrhea and difficulties eating in 3 patients, and facial asymmetry in 2 patients.

The time interval between the onset of facial paralysis and performing the static reanimation procedure was minimum 3 months and maximum 1 year. Surgery was performed under local anesthesia in all cases. 7 patients had multiple procedures, performed either at the same time or in separate sessions. One patient had gold plate insertion into the superior eyelid, lower eyelid ectropion correction and suture suspension of the affected hemiface. Another patient had gold plate insertion into the superior eyelid and inferior eyelid ectropion correction. Five patients had gold plate insertion into the superior eyelid and suture suspension. Two patients had only gold plate insertion into the superior eyelid and another two patients had only suture suspension.

For the patients in which multiple staged procedures were performed, it was considered that the functional results could be improved after the initial facial reanimation surgery, therefore scheduling for another procedure to complete the previous results. After the completion of all procedures, the functional outcomes were optimal, and the aesthetic results were acceptable. Facial asymmetry was ameliorated for all patients that underwent facial suture suspension in all facial segments, while only the upper face was improved in the three patients undergoing only periorbital surgery. Lagophthalmos was improved in all patients with a decrease in the subjective complaints. Sialorrhea and eating difficulties were improved in all patients undergoing suture suspension. There were no infectious complications or hematoma formation postoperatively. Minor ecchymosis and postoperative edema were frequent encounters.

The favorable outcomes are presented through images of a facial nerve paralysis patient that underwent static reanimation by simultaneous gold plate insertion into the superior eyelid and suture suspension (Fig. 1-5).

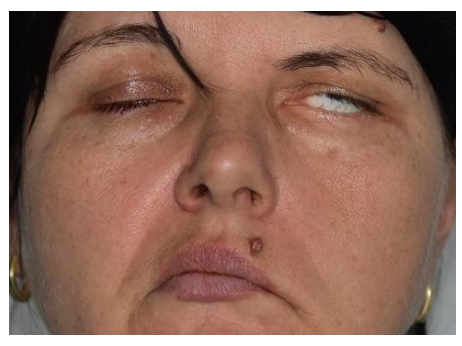

Figure 1. Preoperative aspect of the patient during eye closure, demonstrating left lagophthalmos and facial asymmetry.

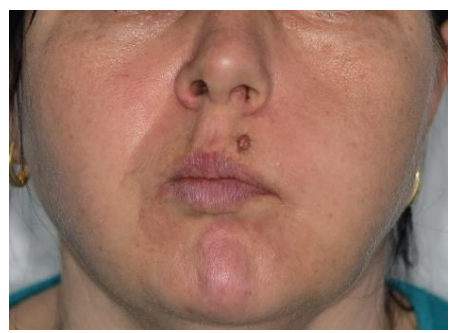

Figure 2. Preoperative aspect of the patient during cheek inflation, showing decreased tonus in the left cheek. 


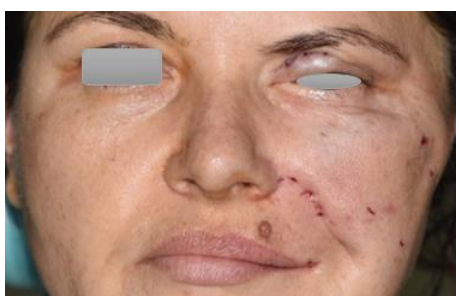

Figure 3. Immediate postoperative aspect following insertion of a gold plate into the left superior eyelid and suture suspension of the left hemiface.

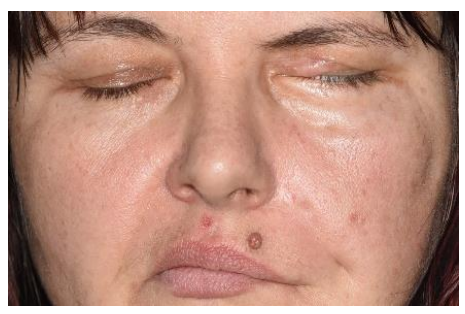

Figure 4. Post-operative aspect 10 days after surgery during eye closure, demonstrating complete left eye closure and ameliorated facial asymmetry with lifting of the left oral commissure, definition of the

nasogenian sulcus and lifting of the cheek with improved position of the inferior eyelid.

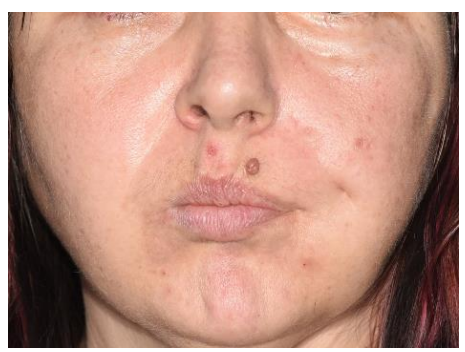

Figure 5. Postoperative aspect 10 days after surgery showing increased tonus of the left cheek and lifted position of the left oral commissure during cheek inflation.

\section{DISCUSSIONS}

Dynamic facial reanimation involves complex, time consuming procedures performed under general anesthesia, that are not without risks. In patients operated for intracranial tumors in which the injured proximal facial nerve cannot be used for primary reconstruction using interposition nerve grafts, a dynamic reanimation may necessitate the scarification of another cranial nerve, including the trigeminal, accessory, hypoglossal or contralateral facial nerve $(1,2)$. The indication for such an invasive procedure should carefully consider the general condition of the patient, the existing associated disorders as well as the long-term prognosis. For older patients with multiple comorbidities, oncologic patients, for patients reluctant to undergo another major procedure or for patients with long-standing facial paralysis, static reanimation offers good outcomes with minimal risks, since it involves local anesthesia and short duration of surgery $(7,8)$.
The goal of static reanimation procedures is to improve the functional and esthetic disturbances associated with facial nerve paralysis. Multiple types of procedures exist, targeting different sequelae of facial paralysis. Often, several procedures are necessary in order to address all issues and achieve best outcomes (9). The good results achieved in our study were due to the careful tailoring of the procedures performed, considering the most bothersome complaint reported by each patient. By addressing the main expressed issue, all patients perceived an improvement after the static reanimation intervention.

The periorbital changes resulting from incomplete eyelid closure and inferior eyelid ectropion are frequently the main focus of reanimation surgery due to the distressing complaints of patients including foreign body sensation and pain, but also due to the serious complications regarding vision that can eventually develop $(10,11)$. In our study, improving eyelid closure was the main goal in the patients primarily having this complaint. In this regard, we achieved optimal outcomes by inserting a gold plate into the superior eyelid, for added weight. Plate positioning was important in order to achieve maximal eyelid closure and avoid plate exposure over time, similarly to the reports of other authors (12). Other existing procedures that can improve lagophthalmos are levator lengthening and palpebral spring insertion (13). However, superior eyelid loading is the preferred method for many surgeons $(14,15)$.

Lagophthalmos is known as the condition that can cause the most functional damage in facial nerve paralysis patients, due to the increased corneal exposure and drying with subsequent ulcerations and infections that can eventually cause blindness or even the loss of the affected eye $(13,16)$. It is frequently the first facial nerve sequelae that is addressed during reanimation procedures. In our study it was also the most prevalent complaint, but in some cases, issues related to difficulties during eating and sialorrhea were reported as more distressing, mainly due to the struggling to eat adequately, but also due to social concerns.

Eating difficulties for facial paralysis patients are mostly caused by the food remaining trapped during mastication in the inferior vestibule that resembles a bag, due to the lack of tonus of the cheek. The associated sialorrhea only worsens the symptoms 
due to mucosa dryness, difficulty in forming the food bolus, but also causing frustration during social encounters. Suspension of the oral commissure and genian region using usual sutures or barbed threads, lifts the tissues, preventing saliva leakage, but also improves the tonus of the cheek, therefore decreasing food retaining $(17,18)$. In addition, lagophthalmos is also improved, since the heavy genian region that pulls down on the inferior eyelid, aggravating ectropion and lagophthalmos, is repositioned superiorly, rendering support to the inferior eyelid $(6,18)$. In our study, in the patients with this main complaint, suture suspension was first performed, since it allowed the initial alleviation of the most distressing symptoms. Ectropion correction was mainly indicated in addition to gold plate insertion, for the patients exhibiting significant laxity and length of the inferior eyelid.

For the patients that were most bothered by the facial asymmetry, suture suspension was also the best initial choice of static reanimation, since it allowed addressing the upper, middle and lower face simultaneously, and therefore ensured better cosmetic results. Good outcomes regarding the restoration of facial symmetry using suspension techniques have also been reported by other studies $(19,20)$. Refinements of particular areas can always be made using barbed sutures, due to the minimally invasive character of the procedure, even when plain suture suspension was initially performed (18).

\section{CONCLUSION}

Static reanimation procedures render favorable results when the type of intervention is tailored to fit the most pressing patient complaint. Particularly for neurosurgical patients with intracranial injury of the facial nerve, these procedures ensure a noninvasive modality of increasing the quality of life of the patients with minimal complications.

\section{REFERENCES}

1. Rozen SM, Harrison BL, Isaacson B, Kutz JW Jr, Roland PS, Blau PA, Barnett SL, Mickey BE. Intracranial Facial Nerve Grafting in the Setting of Skull Base Tumors: Global and Regional Facial Function Analysis and Possible Implications for Facial Reanimation Surgery. Plast Reconstr Surg. 2016; 137(1):267-78.

2. Panossian A. Facial paralysis reconstruction in children and adolescents with central nervous system tumors. J Pediatr Rehabil Med. 2014; 7(4):295-305.

3. Nellis JC, Ishii M, Byrne PJ, Boahene KDO, Dey JK, Ishii LE. Association Among Facial Paralysis, Depression, and Quality of Life in Facial Plastic Surgery Patients. JAMA Facial Plast Surg. 2017; 19(3):190-196.

4. Ishii LE, Nellis JC, Boahene KD, Byrne P, Ishii M. The Importance and Psychology of Facial Expression. Otolaryngol Clin North Am. 2018; 51(6):1011-1017.

5. Robinson MW, Baiungo J. Facial Rehabilitation: Evaluation and Treatment Strategies for the Patient with Facial Palsy. Otolaryngol Clin North Am. 2018; 51(6):1151-1167.

6. Costan W. Management of Extended Parotid Tumors. Editura Springer, 2016; ISBN 978-3-319-26543-8.

7. White $\mathrm{H}$, Rosenthal E. Static and dynamic repairs of facial nerve injuries. Oral Maxillofac Surg Clin North Am. 2013; 25(2):303-12.

8. Kim L, Byrne PJ. Controversies in Contemporary Facial Reanimation. Facial Plast Surg Clin North Am. 2016; 24(3):275-97.

9. Lafer MP, O TM. Management of Long-Standing Flaccid Facial Palsy: Static Approaches to the Brow, Midface, and Lower Lip. Otolaryngol Clin North Am. 2018; 51(6):11411150.

10. Joseph SS, Joseph AW, Douglas RS, Massry GG. Periocular Reconstruction in Patients with Facial Paralysis. Otolaryngol Clin North Am. 2016; 49(2):475-87.

11. Homer N, Fay A. Management of Long-Standing Flaccid Facial Palsy: Periocular Considerations. Otolaryngol Clin North Am. 2018; 51(6):1107-1118.

12. Oh TS, Min K, Song SY, Choi JW, Koh KS. Upper eyelid platinum weight placement for the treatment of paralytic lagophthalmos: A new plane between the inner septum and the levator aponeurosis. Arch Plast Surg. 2018; 45(3):222-228.

13. Leckenby JI, Ghali S, Butler DP, Grobbelaar AO. Reanimation of the brow and eye in facial paralysis: Review of the literature and personal algorithmic approach. J Plast Reconstr Aesthet Surg. 2015; 68(5):60314.

14. Rofagha S, Seiff SR. Long-term results for the use of gold eyelid load weights in the management of facial paralysis. Plast Reconstr Surg 2010;125(1):142e9.

15. Rozen S, Lehrman C. Upper eyelid postseptal weight placement for treatment of paralytic lagophthalmos. Plast Reconstr Surg 2013;131(6):1253e65.

16. Costan V, Doscas A, Ciofu ML, Boişteanu O, Popescu E. On the management of paralytic lagophthalmos. International Journal of Medical Dentistry 2018, 22(4):433-436.

17. Biglioli F. Facial reanimations: part II--long-standing paralyses. Br J Oral Maxillofac Surg. 2015; 53(10):907-12.

18. Costan W, Popescu E, Sulea D, Stratulat IS. A New Indication for Barbed Threads: Static Reanimation of the Paralyzed Face. J Oral Maxillofac Surg. 2018; 76(3):639645.

19. Langille M, Singh P. Static Facial Slings: Approaches to 
Rehabilitation of the Paralyzed Face. Facial Plast Surg Clin North Am. 2016; 24(1):29-35.

20. Leach G, Kurnik N, Joganic J, Joganic E. Multivectored
Superficial Muscular Aponeurotic System Suspension for Facial Paralysis. J Craniofac Surg. 2017; 28(4):882-887. 